\title{
High dimensional quantum entanglement
}

\author{
Sonja Franke-Arnold ${ }^{1, a}$, Alessandra Gatti ${ }^{2,3}$, and Nicolas Treps ${ }^{4}$ \\ 1 SUPA, School of Physics and Astronomy, University of Glasgow, Scotland \\ 2 Istituto di Fotonica e Nanotecnologie del CNR, Milano, Italy \\ 3 Universita degli Studi dell'Insubria, Como, Italy \\ ${ }^{4}$ Université Pierre et Marie Curie, France
}

Received 27 February 2013

Published online 7 May 2013 - (c) EDP Sciences, Società Italiana di Fisica, Springer-Verlag 2013

The phenomenon of quantum entanglement was initially introduced by Einstein and co-workers in their famous EPR paper when questioning the completeness of quantum theory, while the expression "entanglement" was soon after coined by Schrödinger. From an intriguing and fascinating aspect of quantum theory, entanglement has become in the last decades an invaluable resource for the emerging areas of quantum information and quantum technologies.

Fundamental tests of quantum mechanics as well as first applications were, and are, largely based on the two orthogonal polarisation states of light. This well-developed standard approach of quantum optics turns out to be inadequate when confronted by the practical implementation of quantum technologies. Here the imperative is towards faster processing of ever increasing quantities of data, with opportunities provided by employing multi-dimensional and multi-modal quantum entanglement. The generation of entanglement and quantum correlations in high dimensional Hilbert spaces opens the way to a breakthrough in the information capacity of QIPC protocols, and provides a fundamental resource for quantum metrology and quantum imaging. In this context, light plays a fundamental role as the natural carrier of information over large distances and between logic elements within a processor. The longlived matter degrees of freedom instead enable the storage and processing of information. As a result, light-matter interfaces play a crucial role in the development of any quantum communication system.

This special issue focuses on both the experimental and theoretical aspects of high dimensional entanglement involving both light and matter, with an emphasis on the topics addressed by the European FET Open project HIDEAS (High Dimensional Entangled Systems, http://hideas.dfm.uninsubria.it/). The 15 papers of this volume consider the generation and manipulation of high-dimensional quantum entanglement in different context and domains, including

- High-D temporal entanglement of light (quantum frequency combs).

- High-D spatial transverse entanglement of light (orbital angular momentum or linear momentum-position entanglement).

- High-D entanglement of light in mixed domains (space and time).

- Multimode light and matter quantum interfaces.

- Applications of high dimensional entanglement to QIPC protocols and to quantum metrology.

As demonstrated by these papers this is a highly active research field and the developing theories and first proof of principle experiments are highly promising for wider implementation of high dimensional quantum entanglement.

\footnotetext{
a e-mail: sonja.franke-arnold@glasgow.ac.uk
} 